NBER WORKING PAPER SERIES

\title{
RISK TAKING AND GENDER IN HIERARCHIES
}

\author{
Suzanne Scotchmer \\ Working Paper 14464 \\ http://www.nber.org/papers/w14464 \\ NATIONAL BUREAU OF ECONOMIC RESEARCH \\ 1050 Massachusetts Avenue \\ Cambridge, MA 02138 \\ November 2008
}

I thank Larry Blume, David Freedman, and Bob Anderson for guidance in the proof of Lemma 7. I thank an anonymous referee for improving Lemma 1. I thank Bob Anderson, Ian Ayres, Marcus Berliant, Eddie Dekel, Catherine Eckel, Dino Falaschetti, Ed Green, Linda Krieger, David Levine, Anthony Marino, David Neumark, Stephen Maurer, Roberto Raimondo and Joel Sobel for discussion. A previous version of this paper circulated as "Affirmative Action in Hierarchies." I thank the Kauffman Foundation, the Committee on Research of the U.C., Berkeley, Academic Senate, and NSF grant SBE 0830186 for financial support. The views expressed herein are those of the author(s) and do not necessarily reflect the views of the National Bureau of Economic Research.

NBER working papers are circulated for discussion and comment purposes. They have not been peerreviewed or been subject to the review by the NBER Board of Directors that accompanies official NBER publications.

(C) 2008 by Suzanne Scotchmer. All rights reserved. Short sections of text, not to exceed two paragraphs, may be quoted without explicit permission provided that full credit, including $\bigcirc$ notice, is given to the source. 
Risk Taking and Gender in Hierarchies

Suzanne Scotchmer

NBER Working Paper No. 14464

November 2008

JEL No. D63,J08

\begin{abstract}
$\underline{\text { ABSTRACT }}$
In a labor market hierarchy, promotions are affected by the noisiness of information about the candidates. I study the hypothesis that males are more risk taking than females, and its implications for rates of promotion and abilities of survivors. I define promotion hierarchies with and without memory, where memory means that promotion depends on the entire history of success. In both types of hierarchies, the surviving risk takers will have lower average ability whenever they have a higher survival rate. Further, even if more risk takers than non risk takers are promoted in the beginning of the hierarchy, that will be reversed over time. The risk takers will eventually have a lower survival rate, but higher ability. As a consequence of these differences, the various requirements of employment law cannot simultaneously be satisfied. Further, if promotion standards are chosen to maximize profit, the standards will reflect gender in ways that are difficult to distinguish from discriminatory intent.
\end{abstract}

Suzanne Scotchmer

Department of Economics

University of California

Berkeley, CA 94720-7320

and NBER

scotch@berkeley.edu 


\section{Introduction}

In this paper, I study the effect of risk taking on promotion in hierarchies, where promotion at each stage depends on a signal of ability. The motivation comes from a substantial body of evidence that males are more risk taking than females, and from the continuing controversy about why males and females have different patterns of success in labor markets. Granting the premise that the genders differ in risk taking, does this have explanatory power for labor markets? The answer is mixed, partly because the theorems below can be applied to labor markets in different ways.

The theorems proved below compare promotions drawn from two populations, one of which generates accurate signals of ability and the other of which generates noisy signals of ability. The premise is that true abilities have the same distribution in both populations, at least initially, but that agents in one population give a noisy signal to the decision maker. This is a reduced-form hypothesis that might follow from preferences and optimizing behavior, or might reflect behavior that is hard-wired. This distinction does not matter for the theorems that I prove, although it matters for the interpretation.

I introduce two types of promotion hierarchies: those with memory and those without memory. In a hierarchy without memory, promotion at stage $t$ depends only on the signal of ability generated at stage $t$. With memory, promotion can depend on the entire history of signals. Hierarchies such as sports tournaments do not have memory, since survival depends only on winning the current match. Hierarchies such as academic labor markets have memory, although promotion would typically depend more heavily on current performance than on past performance. To emphasize the distinction between hierarchies with memory and those without, I assume for the case of memory that all past signals are used symmetrically. There is no extra weight given to recent performance.

In section 2, I describe a simple model of hierarchical labor markets. In section 3, I show how promotion standards interact with risk taking, and prove an impossibility result related to employment law. Even with one period of promotion, it is impossible to find a promotion policy that simultaneously (a) promotes according to gender-blind standards, (b) promotes 
at equal rates from the two populations, and (c) ensures that the promoted pools have equal expected abilities. The various legal principles about discrimination are in conflict.

In section 4, I study hierarchies with and without memory, focussing on two important statistical properties - survival rates and the expected abilities of survivors. With gender-blind promotion standards, males may be advantaged at the beginning due to their risk-taking behavior, but this will be reversed at the end. Further, any advantage in the promotion rate coincides with a deficit in ability. If males have a higher promotion rate at the beginning, they will have lower average ability than the promoted females, but this will also be reversed at the end.

In section 5, I turn to private objectives rather than social objectives. Different standards will be used in the two cases that (a) promotion means changing employers and (b) all promotions take place within a firm. If a firm only keeps a worker for a single period, then the promotion standards that materialize will equalize the marginal abilities of promoted workers in the two populations. But if promotions are within a firm, the firm has an incentive to preserve options on the employees with noisy signals, since more information will be collected later. At early stages, the firm will retain risk takers who have lower expected ability than is required of the non risk takers. If the males are the risk takers, males are favored.

Since the paper is motivated by the considerable evidence that males are more risk taking than females, ${ }^{1}$ I will refer to risk takers as males, and non risk takers as females. However, although the males' risk taking may lead to noise in formal labor-market signals like exams, there are other social phenomena that may cause females' signals to be noisier than those of males. Promotions often rely on informal evidence like letters of reference. If males are observed more closely than females, then it may be females who have noisier signals. Informal evidence is often under the control of the ob-

\footnotetext{
${ }^{1}$ See Eckel and Grossman (2002) on gambling behavior, Bajtesmit and Bernasek (1996), Jianakoplos and Bernasek (1998) and Charness and Gneezy (2004) on investment behavior, Slovic (1966), Mittal and Dhade (2007), and Ginsburg and Miller (1982) for experiments with children, and Espinosa and Gardeazabal (2005) on gender differences in exam strategies. See Eckel and Grossman (2005a) for a comparison of the results from field studies versus contextual environmental experiments. See Dekel and Scotchmer (1999) for an evolutionary argument for the gender difference.
} 
server rather than the candidate. An academic in hope of promotion cannot commandeer attendance at a seminar, and an employee cannot demonstrate competence without being given a task. If opportunities to give informal evidence are more available to males than females, then the signals generated by males may be more accurate than those of females, and we must reverse our understanding of the theorems in this paper.

\section{Risk Takers, Non Risk Takers, and Hierar- chies}

Suppose that agents' abilities are a random variable $A$ with distribution $G$ and density $g$, finite variance, and support equal to the real line. Males and females have the same distribution of abilities. A male with ability $A=a$ generates a sequence of independent random signals $Z_{1}, Z_{2}, \ldots Z_{t}, \ldots$ each with distribution $\Phi(\cdot \mid a)$, density $\phi(\cdot \mid a)$, mean $a$, finite variance, and support equal to the real line.

I assume throughout that the monotone likelihood ratio property holds:

$$
\frac{\phi\left(z_{1} \mid a_{1}\right)}{\phi\left(z_{1} \mid a_{2}\right)}>\frac{\phi\left(z_{2} \mid a_{1}\right)}{\phi\left(z_{2} \mid a_{2}\right)} \text { when } z_{1}>z_{2} \text { and } a_{1}>a_{2}
$$

I sometimes use the following more specific assumption that the signal $Z$ is ability plus noise.

Assumption $A$ : Conditional on $A=a$, for each $t$, the random variable $Z_{t}$ is equal to $a+U_{t}$, where $U_{1}, U_{2}, \ldots U_{t}, \ldots$ are distributed independently with density $\phi^{u}$, and $\phi(z \mid a)=\phi^{u}(z-a)$. The densities $g$ and $\phi^{u}$ are single peaked and symmetric around 0 , with finite variance and supports equal to the real line.

Promotion standards are a sequence of real numbers, $c=c_{1}, c_{2}, \ldots c_{t}, \ldots$ interpreted as thresholds for promotion. Due to the monotone likelihood ratio, the threshold policy selects agents that have higher ability in expectation.

Say that the promotion standards are gender blind if all agents face the same promotion standards. When I do not assume gender-blind pro- 
motion standards, I will refer to the males' promotion standards by $m=$ $m_{1}, m_{2}, \ldots m_{t}, \ldots$ and to the females' promotion standards by $f=f_{1}, f_{2}, \ldots f_{t}, \ldots$

Say that the promotion standards $c$ are bounded if there exist $\underline{c}, \bar{c}$ such that $\underline{c} \leq c_{t} \leq \bar{c}$ for all $t$.

A female with ability $a$ survives to stage $t$ if $a \geq c_{d}$ for each $d=1, \ldots t$. The probability of survival $\mathcal{S}_{t}^{F}(a, c)$ is directly an indicator function:

$$
\mathcal{S}_{t}^{F}(a, c)= \begin{cases}0 & \text { if } a<c_{d} \text { for some } d \leq t . \\ 1 & \text { if } a \geq c_{d} \text { for each } d \leq t .\end{cases}
$$

The survival rate of females at stage $t$ is

$$
E\left[\mathcal{S}_{t}^{F}(A, c)\right]=\int_{-\infty}^{\infty} \mathcal{S}_{t}^{F}(a, c) g(a) d a=1-G\left(\max _{d \leq t}\left\{c_{d}\right\}\right)
$$

The expected ability of a random female survivor is

$$
E^{F}[A \mid c, t]=\frac{1}{E\left[\mathcal{S}_{t}^{F}(A, c)\right]} \int_{\max _{d \leq t}\left\{c_{d}\right\}}^{\infty} a g(a) d a
$$

To define the survival rate of males, I distinguish between promotion hierarchies that have memory and those that do not. In a hierarchy without memory, promotion at stage $t$, conditional on survival to $t-1$, depends only on the performance in period $t$. In a hierarchy with memory, promotion at stage $t$, conditional on survival to $t-1$, depends on the cumulative performance to $t$. I will study the special case in which promotion depends symmetrically on all the previous signals, through the mean.

\subsection{Survival in hierarchies without memory}

Say that a male survives to $t$ in a hierarchy without memory if $Z_{d} \geq c_{d}$, for $d=1, \ldots t$. For a male with ability $A=a$, denote a male's probability of survival by $\mathcal{S}_{t}^{M}(a, c)$ :

$$
\mathcal{S}_{t}^{M}(a, c)=\Pi_{d=1}^{t}\left(1-\Phi\left(c_{d} \mid a\right)\right)
$$


The survival rate of males at stage $t$ (without memory) is

$E\left[\mathcal{S}_{t}^{M}(A, c)\right]=\int_{-\infty}^{\infty} \mathcal{S}_{t}^{M}(a, c) g(a) d a=\int_{-\infty}^{\infty} g(a) \Pi_{d=1}^{t}\left[1-\Phi\left(c_{d} \mid a\right)\right] d a$

The expected ability of a random male survivor at stage $t$ is

$$
E^{M}[A \mid c, t]=\frac{E\left[A \mathcal{S}_{t}^{M}(A, c)\right]}{E\left[\mathcal{S}_{t}^{M}(A, c)\right]}=\int_{-\infty}^{\infty} a \frac{g(a) \Pi_{d=1}^{t}\left[1-\Phi\left(c_{d} \mid a\right)\right]}{\int_{-\infty}^{\infty} g(a) \Pi_{d=1}^{t}\left[1-\Phi\left(c_{d} \mid a\right)\right] d a} d a
$$

A marginal survivor at stage $t$ is an agent who generates signal $Z_{t}=c_{t}$. The expected ability of a marginal male survivor is

$$
e^{M}[A \mid c, t]=\int_{-\infty}^{\infty} a \frac{g(a) \phi\left(c_{t} \mid a\right) \mathcal{S}_{t-1}^{M}(a, c)}{\int_{-\infty}^{\infty} g(a) \phi\left(c_{t} \mid a\right) \mathcal{S}_{t-1}^{M}(a, c) d a} d a
$$

\subsection{Survival in hierarchies with memory}

For the random sequence $Z_{1}, Z_{2}, \ldots Z_{t}, \ldots$ define the sequence of sample means $\bar{Z}^{1}, \bar{Z}^{2}, \ldots \bar{Z}^{t}, \ldots$ where $\bar{Z}^{t}=\frac{1}{t} \sum_{k=1}^{t} Z_{k}$ for each $t$. Say that a male survives to $t$ in a hierarchy with memory if $\bar{Z}^{d} \geq c_{d}$, for $d=1, \ldots$.

When $A=a$, denote the probability of survival to $t$ by $\overline{\mathcal{S}}_{t}^{M}(a, c)$, which can be written as

$$
\overline{\mathcal{S}}_{t}^{M}(a, c)=\int_{c_{1}}^{\infty} \phi\left(z_{1} \mid a\right) \int_{2 c_{2}-z_{1}}^{\infty} \phi\left(z_{2} \mid a\right) \ldots \int_{t c_{t}-\sum_{k=1}^{t-1} z_{k}}^{\infty} \phi\left(z_{t} \mid a\right) d z_{t} \ldots d z_{2} d z_{1}
$$

The survival rate of males at stage $t$ (with memory) is $E\left[\overline{\mathcal{S}}_{t}^{M}(A, c)\right]$.

The expected ability of a random male survivor at stage $t$ is

$$
\bar{E}^{M}[A \mid c, t]=\frac{E\left[A \overline{\mathcal{S}}_{t}^{M}(A, c)\right]}{E\left[\overline{\mathcal{S}}_{t}^{M}(A, c)\right]}=\int_{-\infty}^{\infty} a \frac{\overline{\mathcal{S}}_{t}^{M}(a, c) g(a)}{\int_{-\infty}^{\infty} \overline{\mathcal{S}}_{t}^{M}(a, c) g(a) d a} d a
$$

A marginal survivor at stage $t$ is an agent who generates signal $\bar{Z}^{t}=c_{t}$. With memory, the probability of being marginal at stage $t$ depends not only 
on the stage- $t$ signal $Z_{t}$, but on the accumulated success up to that point, $\sum_{k=1}^{t-1} Z_{k}$. Let $\bar{g}(\cdot \mid t, c)$ be the probability density describing the distribution of ability, conditional on surviving to $t-1$ and being marginal at $t$. Then

$$
\begin{aligned}
& \bar{g}(a \mid t, c)= \\
& \frac{g(a) \int_{c_{1}}^{\infty} \phi\left(z_{1} \mid a\right) \ldots \int_{(t-1) c_{t}-\sum_{k=1}^{t-2} z_{k}}^{\infty} \phi\left(z_{t-1} \mid a\right) \phi\left(t c_{t}-\sum_{k=1}^{t-1} z_{k} \mid a\right) d z_{t-1} . . d z_{1}}{\int_{-\infty}^{\infty} g(a)\left[\int_{c_{1}}^{\infty} \phi\left(z_{1} \mid a\right) \ldots \int_{(t-1) c_{t}-\sum_{k=1}^{t-2} z_{k}}^{\infty} \phi\left(z_{t-1} \mid a\right) \phi\left(t c_{t}-\sum_{k=1}^{t-1} z_{k} \mid a\right) d z_{t-1} . . d z_{1}\right] d a}
\end{aligned}
$$

The expected ability of a marginal survivor is given by

$$
\bar{e}^{M}[A \mid c, t]=\int_{-\infty}^{\infty} a \bar{g}(a \mid c, t) d a
$$

At each $a$, the probability of survival, $\overline{\mathcal{S}}_{t}^{M}(a, c)$, is decreasing with $t$, and bounded below by zero. Hence the sequence converges at each $a$. Let

$$
\overline{\mathcal{S}}^{M}(a, c)=\lim _{t \rightarrow \infty} \overline{\mathcal{S}}_{t}^{M}(a, c) \text { for each } a \in \mathbf{R}
$$

In a hierarchy with memory, the limit distribution of surviving risk takers has density defined by the following.

$$
\frac{g(a) \overline{\mathcal{S}}^{M}(a, c)}{\int_{-\infty}^{\infty} g(a) \overline{\mathcal{S}}^{M}(a, c) d a}
$$

I show in Lemma 7 below that the support is $[\bar{c}, \infty)$, where $\bar{c}=\lim \sup c_{t}$.

Because the survival function $\overline{\mathcal{S}}_{t}^{M}(\cdot, c)$ is increasing, the limit function $\overline{\mathcal{S}}^{M}(\cdot, c)$ is nondecreasing.

\section{Antidiscrimination goals: an impossibility result}

Before turning to how promotion plays out in a hierarchy, I use a single round of promotion to show how differences in risk taking create a conflict among the following three natural objectives of labor policy: 


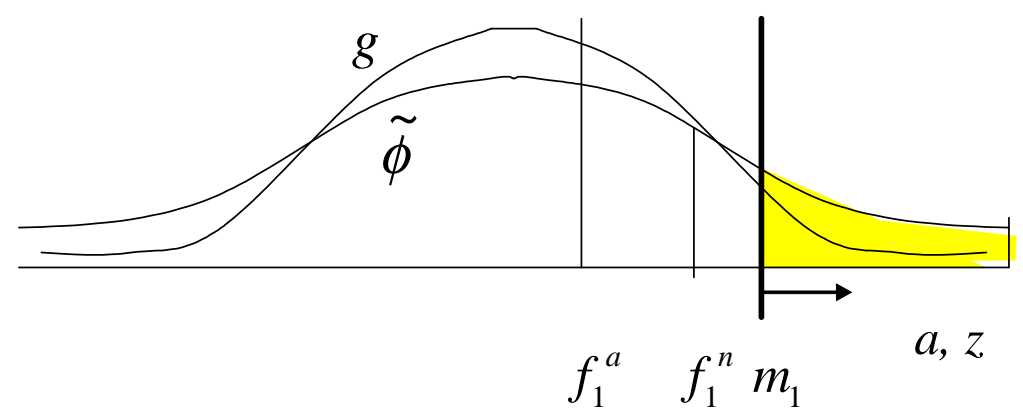

Figure 1: First stage of the hierarchy

- gender-blind promotion standards

- equal survival rates

- equal abilities of survivors.

In figure 1, the distribution of abilities $A$ (hence the distribution of signals generated by females) is shown by the density $g$. For this purpose, suppose that Assumption A holds. Then the distribution of the signal $Z$ generated by males is a mean-preserving spread of $g$. The marginal distribution of $Z$ is represented by the density $\tilde{\phi}$ in figure 1 .

Suppose the males' promotion standard at stage 1 is $m_{1}$. The resulting probability of survival is the area to the right of $m_{1}$. If the promotion standard is gender blind, all females with signal (ability) above $m_{1}$ are also promoted. However, there will be fewer females than males, provided $m_{1}>0$, since $\tilde{\phi}$ is a mean-preserving spread of $g$.

At the same time, females who exceed the standard $m_{1}$ have higher average ability than males. This follows from a very general result, stated in Lemma 1 below, that the average ability of surviving males is lower than the average ability of surviving females in any period where males have a higher survival rate. We thus see immediately - and it is stated formally in Proposition 2 - that gender-blind standards are incompatible with equal promotion rates and with equal abilities. To equalize the survival rates, the female promotion standard must be lower, namely, $f_{1}^{n}$ in figure 1 , and to 
equalize abilities, the female promotion standard must be lower still, namely $f_{1}^{a}$.

That the promotion standards are ordered as drawn is stated in Proposition 2 .

Lemma 1 [If male have a higher survival rate than females, males have lower average ability.] Let $(m, f)$ be promotion standards for males and females. Suppose that the survival rate of males is no smaller than the survival rate of females at time $t$ :

$$
\begin{gathered}
E\left[\mathcal{S}_{t}^{M}(A, m)\right] \geq E\left[\mathcal{S}_{t}^{F}(A, f)\right] \\
\text { respectively, } E\left[\overline{\mathcal{S}}_{t}^{M}(A, m)\right] \geq E\left[\mathcal{S}_{t}^{F}(A, f)\right]
\end{gathered}
$$

Then the expected ability of a random surviving male is smaller than the expected ability of a random surviving female:

$$
\begin{aligned}
E^{M}[A \mid m, t] & <E^{F}[A \mid f, t] \\
\text { respectively, } \bar{E}^{M}[A \mid m, t] & <E^{F}[A \mid f, t]
\end{aligned}
$$

The lemma is proved in the appendix. It uses essentially no assumptions on the distributions or promotion standards, and is thus very general. The intuition is simply that, because males generate noisy signals, mistakes are made in promoting them. Some high ability males are not promoted, and some low ability males are promoted. In contrast, no mistakes are made in promoting females. Hence, if males and females are promoted in equal numbers, the average ability of the surviving males must be lower.

Proposition 2 [The impossibility of equalizing survival rates, abilities and standards] Let $m$ be the promotion standards for males. Let $f^{n}$ (respectively $f^{a}$ ) be promotion standards for females such that their survival rate (respectively, ability) equals that of surviving males at stage 1. Then

(a) $f_{1}^{a}<f_{1}^{n}$.

(b) If assumption $A$ holds and $m_{1}>0$, then $f_{1}^{n}<m_{1}$. 
Proof Part (a) follows because $E\left[\mathcal{S}_{1}^{F}\left(A, f^{n}\right)\right]=E\left[\mathcal{S}_{1}^{M}(A, m)\right]<E\left[\mathcal{S}_{1}^{F}\left(A, f^{a}\right)\right]$. The first equality is by definition of $f^{n}$, and the second follows from Lemma 1. If $E\left[\mathcal{S}_{1}^{M}(A, m)\right] \geq E\left[\mathcal{S}_{1}^{F}\left(A, f^{a}\right)\right]$ then the expected ability of male survivors would be smaller than that of females at stage 1 , a contradiction.

(b) Using Assumption A, the distribution of ability second-order dominates the distribution of the signal $Z$. Both have mean 0 , so for $m_{1}>0$, the probability that a male survives (namely, the probability that $Z>m_{1}$ ) exceeds the probability that a female survives according to the same standard (the probability that $A>m_{1}$ ). Hence, to increase the probability that a female survives, and in particular to equalize the survival rates, it must hold that $f_{1}^{n}<m_{1}$.

Proposition 2 illustrates why "affirmative action" in labor markets is a vexed issue. It is not possible at the same time to equalize numbers, equalize abilities, and also to satisfy the procedural objective of having gender-blind standards. $^{2}$

However, employment law seems to have all three objectives. Antidiscrimination law in the U.S. dates mainly to Title VII of the 1964 Civil Rights Act, and its extension in the 1972 Equal Employment Opportunity Act. ${ }^{3}$ These Acts disallow disparate treatment by employers based on race, color, sex, national origin, or religion, with respect to hiring, promotion, compensation, or termination decisions. ${ }^{4}$ At the same time, an employer' practices might run afoul of Title VII if they have a disparate impact on a protected group. ${ }^{5}$

\footnotetext{
${ }^{2}$ Spitzer (1979) makes a similar argument, showing how courts and agencies must balance objectives which can be mutually inconsistent. Most pertinently, he applies his analysis to the Bakke decision in which the split decision of the Supreme Court is largely due to the fact that different justices were concerned with different objectives, all legitimate, which cannot simultaneously be satisified.

${ }^{3}$ A great deal has been written on the legal details and their impact. On the economic side, see Rodgers (2006). The chapter by Holzer and Neumark outlines the broad contours of the law. For a history of how the law developed, see Blumrosen (1993), and for detail on the evidentiary conundrums that arise, see, for example, Krieger (1995).

${ }^{4}$ The more pro-active notion of "affirmative action" originates in Executive Orders 10925 (1961), 11246 (1965,1967), 11625 (1971) of the Kennedy, Johnson, and Nixon administrations, respectively, aimed at federal contractors.

${ }^{5}$ The ban on disparate impacts evolved from policies of the Equal Employment Opportunity Commission (EEOC), which was created to enforce the Civil Rights Act. See Blumrosen (1993), 73-75 and Leiter and Leiter (2002), 43-47. In the absence of clear
} 
Whereas disparate treatment concerns the individual, and in particular, whether the employer intended to discriminate against the individual, disparate impact is about the effects of an employment practice on an entire group. The employer's intent is not at issue in disparate impact cases. A Title VII disparate-impact violation results when a seemingly neutral employment practice affects opportunities in ways that are not race- or gender-neutral, and where the employer cannot prove that the practice is justified under Title VII's business necessity defense. ${ }^{6}$

Proposition 2 points out that the bans on disparate treatment and disparate impact are contradictory. Title VII insists on gender-blind employment practices, but gender-blind employment practices lead to disparate impacts on the numbers promoted and on survivors' abilities. These disparate impacts cannot be remedied by gender-blind promotion standards. Under the conditions of Proposition 2, the standards for females must be lower than for males early in the hierarchy, but (as we will see) higher as the hierarchy progresses.

The contradiction between gender- or race-blindness and disparate impact surfaces in personnel policies that seem incomprehensible. ${ }^{7} \mathrm{MIT}^{\text {'s } \mathrm{Hu}-}$ man Resources page ${ }^{8}$ poses the question "Is affirmative action an employment quota system?" Since "quotas are expressly forbidden by federal law," the answer is that if "underutilization" of minority groups is discovered, ... "placement goals are established .. Goals are targets, not quotas." The page

definitions or a clear legislative intent behind the Civil Rights Act, the EEOC pursued policies that imputed to the law an ambition to improve the economic circumstances of minorities. This led to policies against disparate impact that were enunciated as law by the Supreme Court in Griggs.

${ }^{6}$ Krieger (1995) discusses why it is difficult to prove discrimination under both theories. She argues that disparate treatment may result from subtle stereotypes even where there is no discriminatory intent. Where there are patterns and practices of discrimination, also known as systemic disparate treatment, she argues that disparate impact theory is like a software "patch," (p. 1227). Although disparate impact theory circumvents the intent requirement, it creates problems that make judges reluctant to apply it. Moreover, Krieger argues that, in response to disparate impact theory, employers have shifted from quantifiable criteria like test scores to subjective standards involving unquantifiable variables.

${ }^{7}$ That contradictions arise from competing values has been convincingly discussed in the case of race by Edley (1996).

${ }^{8} \mathrm{http}: / /$ web.mit.edu/hr/aa/what_is.html 
does not explain the difference between targets and quotas, but asserts that there is no conflict between targets and "principles of merit." Proposition 2 suggests otherwise.

\section{The hierarchies}

I now turn to what happens as the hierarchy continues after the first stage. In particular, I assume there are infinitely many periods of promotion. The infinite sequence is unrealistic, but reveals a pattern for late stages that might otherwise be obscure. In particular, with gender-blind standards, there is a reversal in the relative numbers and relative abilities of the surviving populations of males and females. At the beginning of the hierarchy, under the reasonable conditions of figure 1, gender-blind strategies lead to a higher survival rate among males than females, but lower ability. At the end, this is reversed. I show that the reversal occurs in hierarchies with and without memory.

\subsection{The hierarchy without memory}

I first discuss how the hierarchy without memory plays out. Proposition 4 says that, in a hierarchy with gender blind standards, there is eventually a higher survival rate among females, but they have lower ability. This is essentially because males have an opportunity to throw themselves out of the pool at every stage, and will eventually do so, except possibly those with very high ability.

Lemma 3 is proved in the appendix.

Lemma 3 [Only males with very high ability survive in the limit.] Let $m=m_{1}, m_{2}, \ldots m_{t}, \ldots$ be bounded promotion standards for a hierarchy without memory. Then

(a) Given $\varepsilon>0$, there exists $\tilde{t}$ such that for $t>\tilde{t}$, the survival rate (3) of males at stage $t$ is less than $\varepsilon$. 
(b) Given $\zeta \in(0,1)$ and $x>0$, there exists $\tilde{t}$ such that for $t>\tilde{t}$, the probability is smaller than $\zeta$ that a male survivor has ability less than $x$.

(c) Given $x>0$, there exists $\tilde{t}$ such that for $t>\tilde{t}$, the expected ability (4) of a male survivor is larger than $x$.

(d) Given $x>0$, there exists $\tilde{t}$ such that for $t>\tilde{t}$, the expected ability (5) of a marginal male survivor is larger than $x$.

Proposition 4 [Gender-blind promotions without memory] Let $c=c_{1}, c_{2}, \ldots c_{t}, \ldots$ be bounded, gender-blind promotion standards for a hierarchy without memory. Then there exists $\tilde{t}$ such that for $t>\tilde{t}$, the survival rate of males is smaller than the survival rate of females, but the surviving males have higher expected ability.

The proposition follows from Lemma 3. That males eventually survive with smaller probability than females follows directly from Lemma 3(a), by choosing $\varepsilon>0$ such that $\left(1-G\left(c_{t}\right)\right)>\varepsilon$ for all $t$. That male survivors eventually have higher expected ability than surviving females follows by choosing $x$ in Lemma 3(c) to be larger than the (bounded) expected ability of surviving females.

Now suppose that the survival rates are equal rather than that standards are gender blind. Then Proposition 5 says that the males' promotion standards cannot be bounded below. This can be interpreted to mean that promotion standards become irrelevant; all surviving males are eventually promoted.

Since the promotion standards for females are bounded, there will eventually be a stationary, positive measure of surviving females. Hence, the population of surviving males must also become stationary with a positive measure of survivors. But if the males' promotion standards are bounded below, males still have an opportunity to throw themselves out of the pool at every stage, and their numbers will decline. To avoid this, the promotion standards must become very low in order to keep all the males in the pool. Males appear to be getting special treatment, but no males are profiting from the special treatment because their performance is well above the very low standard. 
Proposition 5 [Equal survival rates in a hierarchy without memory] Let $(m, f)$ be promotion standards in a hierarchy without memory, such that males and females have the same survival rates at each stage $t$. If the promotion standards $f$ are bounded, then the performance standards $m$ are not bounded below.

Proof Since $f_{1}, f_{2}, \ldots f_{t}, \ldots$ converge to a finite number, the female survival rates $E\left[\mathcal{S}_{t}^{F}(A, f)\right], t=1,2, \ldots$, converge to a positive number, say $L>$ 0 , and, by hypothesis, the male survival rates $E\left[\mathcal{S}_{t}^{M}(A, m)\right], t=1,2, \ldots$, converge to the same limit, $L$. Suppose, contrary to the proposition, that the sequence $m$ is bounded below by $\underline{m}$. Since $\left(1-\Phi\left(m_{t} \mid a\right)\right) \leq(1-\Phi(\underline{m} \mid a))$ for all $a$, the male survival rate at stage $t$ satisfies

$$
E\left[\mathcal{S}_{t}^{M}(A, m)\right]=\int_{-\infty}^{\infty} g(a) \mathcal{S}_{t}^{M}(a, m) d a \leq \int_{-\infty}^{\infty} g(a)(1-\Phi(\underline{m} \mid a))^{t} d a
$$

Choose an $\varepsilon>0$ such that $\varepsilon<L$. Choose $\tilde{a}$, $\hat{a}$ such that $\hat{a}<\tilde{a}$ and

$$
\begin{aligned}
1-G(\tilde{a}) & <\varepsilon / 3 \\
G(\hat{a}) & <\varepsilon / 3
\end{aligned}
$$

Due to the monotone likelihood ratio property, $\Phi(\underline{m} \mid a)$ decreases with $a$. Choose $\hat{t}$ such that $(1-\Phi(\underline{m} \mid \tilde{a}))^{\hat{t}}<\varepsilon / 3$. Then if $t>\hat{t}$, the upper bound on the male survival rate (10) at stage $t$, satisfies

$$
\begin{aligned}
& \int_{-\infty}^{\hat{a}} g(a)(1-\Phi(\underline{m} \mid a))^{t} d a+\int_{\hat{a}}^{\tilde{a}} g(a)(1-\Phi(\underline{m} \mid a))^{t} d a+\int_{\tilde{a}}^{\infty} g(a)(1-\Phi(\underline{m} \mid a))^{t} d a \\
&<\int_{-\infty}^{\hat{a}} g(a) d a+[G(\tilde{a})-G(\hat{a})](1-\Phi(\underline{m} \mid \tilde{a}))^{t}+\int_{\tilde{a}}^{\infty} g(a) d a \\
&<\varepsilon / 3+(1-\Phi(\underline{m} \mid \tilde{a}))^{t}+\varepsilon / 3<\varepsilon<L
\end{aligned}
$$

This is a contradiction.

Proposition 6 [Equal ability in a hierarchy without memory] 
(a) Suppose, in a hierarchy without memory, that the expected abilities of surviving males and females are equal at stage $\hat{t}$ under the promotion standards $(m, f)$. Then the female survival rate at stage $\hat{t}$ is greater than the male survival rate.

(b) In a hierarchy without memory, there are no bounded promotion standards $(m, f)$ for which surviving males have the same average ability as surviving females at each stage, $t$.

Proposition 6(a) follows directly from Lemma 1, which would otherwise be contradicted. Proposition 6(b) follows from Lemma 3(c) by choosing $x$ larger than the expected ability of female survivors at every $t$.

\subsection{The Hierarchy with Memory}

I now show that, if the hierarchy has memory, males survive in the limit with positive probability. As the hierarchy progresses, it is increasingly difficult to overturn a survivor's accumulated success. Each new signal is blended into the average, and its effect is therefore muted. Nevertheless, if the standards are gender blind, it is still true that eventually a smaller proportion of males than females survives, and they will have higher average ability. This must be proved in a different manner than the analogous result without memory (Proposition 4), since the analog to Lemma 3 does not hold.

Proposition 8 follows from the shape of the limiting survival function $\overline{\mathcal{S}}^{M}(\cdot, c)$, described in Lemma 7 and shown in figure 2. The proof is in the appendix.

Lemma 7 [The limit distribution in a hierarchy with memory] Suppose that the promotion standards are $c=c_{1}, c_{2} \ldots c_{t}, \ldots$, and $\lim \sup c_{t}=\bar{c}$. For each $a \leq \bar{c}, \overline{\mathcal{S}}^{M}(a, c)=0$. Given $\varepsilon>0$, for each $a>\bar{c}+\varepsilon, \overline{\mathcal{S}}^{M}(a, c)>0$.

Proposition 8 uses the hypothesis that promotion standards are nondecreasing. With decreasing promotion standards, all females with ability above $c_{1}$ would survive, and none would be eliminated after stage one. This 


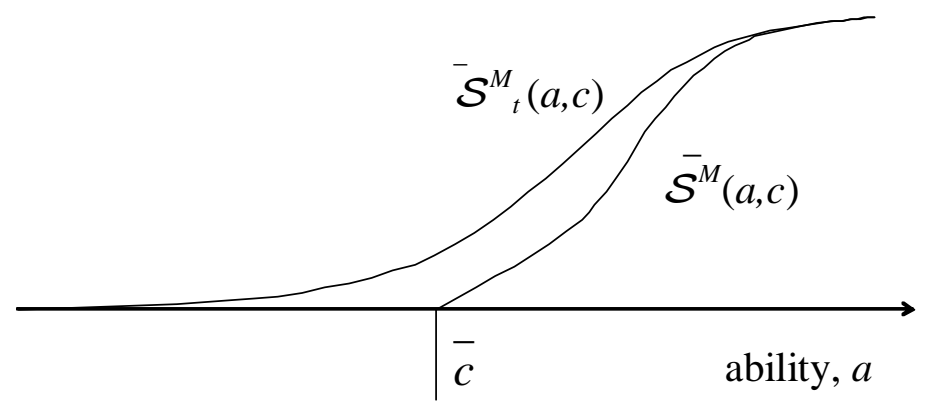

Figure 2: Survival in a Hierarchy with Memory

is not true of males. If the performance standards decrease rapidly, males with abilities lower than $c_{1}$ might survive in large numbers, and the limiting expected ability of males could be lower than that of females. In any case, increasing promotion standards are the more natural case.

Proposition 8 [Gender-blind promotions with memory] Let $c=c_{1}, c_{2} \ldots c_{t}, \ldots$ be nondecreasing, gender-blind promotion standards that converge to $\bar{c}$ in a hierarchy with memory. Then there exists $\hat{t}$ such that for $t>\hat{t}$, the survival rate of males is smaller than that of females, but the surviving males have higher expected ability.

Proof Using Lemma 7, for $a<\bar{c}$, neither males nor females survive. For $a>\bar{c}$, the probability that a female survives is one, while, for males, the survival probability is less than one: $\overline{\mathcal{S}}^{M}(a, c)<1-\Phi\left(c_{1} \mid a\right)<1$. Thus, the limit survival rate of males is smaller than that of females.

Because the males' limit probabilities of survival are nondecreasing with $a$, and strictly increasing on some domain (since $\overline{\mathcal{S}}^{M}(a, c)>\overline{\mathcal{S}}^{M}(\bar{c}, c)$ for some $a>c$ ), the limit distribution of their abilities first-order dominates the 
limit distribution of females' abilities. Thus, the expected ability of surviving males is greater than that of surviving females.

Proposition 9 [Equal average ability with memory] Suppose the hierarchy has memory, and suppose that $(m, f)$ are promotion standards such that the expected abilities of a random surviving male and a random surviving female are equal at stage $t$. Then the survival rate of females at stage $t$ is greater than that of males.

Proposition 9 follows directly from Lemma 1.

\section{Private Objective Functions}

So far, the strategy of this paper has been to study the promotion standards that satisfy some social objective, such as equal survival rates of males and females or gender-blind standards, and to show the conflicts that are created. I now characterize the promotion standards that will arise without legal constraints. I will not start from the premise that employers dislike diversity, or that they are willing to sacrifice profit in order to avoid it, but rather from the premise that employers are profit maximizers whose decisions may nevertheless lead to disparate impacts. The objective is to see which of the social goals are preserved, if any, and whether "discrimination" can be identified. $^{9}$

The implicit assumption in what follows is that wages can be different at different stages of the hierarchy, but wages cannot be different for males and females, and wages do not depend directly on signals.

\footnotetext{
${ }^{9}$ The conceit in this paper is that promotion standards are set according to some well conceived objective, either constrained by law, or guided by profit, but not guided by reference to the pool that is already promoted. For an interesting discussion of the latter, see Sobel (2001).
} 


\subsection{Promotions across firms}

Suppose that, to be promoted, an agent must get a job with a different employer. An employer hiring at $t$ selects from the survivors to $t-1$. This creates externalities among firms, since the distribution of abilities in an employer's pool is determined by previous employers' promotion decisions.

Because an employer retains its workers for a single period, its sole objective is to take the workers with highest ability. For each $t=1,2, \ldots$, let $W_{t}$ be the profit objective when the industry does not have memory, and let $\bar{W}_{t}$ be the objective when the industry has memory:

$$
\begin{aligned}
W_{t}(m, f) & = \\
& \int_{-\infty}^{\infty}\left(a-w_{t}\right) \mathcal{S}_{t}^{M}(a, m) g(a) d a+\int_{f_{t}}^{\infty}\left(a-w_{t}\right) g(a) d a \\
\bar{W}_{t}(m, f) & = \\
& \int_{-\infty}^{\infty}\left(a-w_{t}\right) \overline{\mathcal{S}}_{t}^{M}(a, m) g(a) d a+\int_{f_{t}}^{\infty}\left(a-w_{t}\right) g(a) d a
\end{aligned}
$$

The profit function is an aggregate for all employers at stage $t$. The wage rate is $w_{t}$, and ability $a$ is understood as the profit of employing an agent with ability $a$. I assume that the wages are bounded and nondecreasing, and converge to $\bar{w}$. This will imply that the female promotion standards $f$ are bounded and nondecreasing, and also converge. ${ }^{10}$

It is straightforward to show that the optimal standards equalize the expected abilities of marginal survivors:

Lemma 10 If the promotion standards $(m, f)$ maximize total ability net of wages, (11a) (respectively, (11b)), then the standards $\left(m_{t}, f_{t}\right)$ equalize the abilities of marginal males and marginal females at $t$. In particular, (12a) (respectively, (12b)) holds.

$$
\begin{aligned}
& e^{M}[A \mid m, t]-w_{t}=f_{t}-w_{t}=0 \\
& \bar{e}^{M}[A \mid m, t]-w_{t}=f_{t}-w_{t}=0
\end{aligned}
$$

\footnotetext{
${ }^{10} \mathrm{In}(11 \mathrm{a})$ and $(11 \mathrm{~b})$, that is why I wrote the objective function for females with a minimum ability $f_{t}$ rather than $\max _{d \leq t}\left\{f_{d}\right\}$.
} 
Proposition 11 characterizes the standards that will arise endogenously when promotions are across firms. Males will be held to a higher standard at the earliest stage provided fewer than half are promoted, but in the hierarchy without memory, males will eventually be promoted on weak performance, much weaker than required of females. This is for a reason similar to when standards are chosen to equalize promotion rates (Proposition 5). If the males' promotion standards did not become low, males would keep throwing themselves out of the pool, and the expected ability of the marginal (and average) survivor would keep rising. But if the objective is to stop them from getting thrown out of the pool, and hence to equalize the abilities of marginal survivors, then the males' standard must become low. A male's probability of generating such a low standard must go to zero.

For the hierarchy with memory, I will use Assumption B in order to avoid complicated arguments based on rates of convergence.

Assumption B: In the limit of the hierarchy with memory, each male's ability is known, and the distribution of males' abilities has the limit distribution (7).

Since the abilities of males are revealed in the limit, their standard for promotion will ultimately be the same as for females. There will be marginal females in the limit, in the sense that there remain a positive mass of females with abilities in a neighborhood of the limit promotion standard, $\bar{f}$. However, due to the selection process before reaching the limit, there will be no marginal males. Proposition 11(b),(c) say that the probability of a risk-taker being marginal at late stages of the hierarchy converges to zero.

In the hierarchy with memory, the marginal probability density of the signal $Z_{t}$ at stage $t$ is given by

$$
\tilde{\phi}_{t}(z)=\int_{-\infty}^{\infty} \phi(z \mid a) \frac{g(a) S_{t}^{M}(a, m)}{\int_{-\infty}^{\infty} g(a) S_{t}^{M}(a, m) d a} d a
$$

Proposition 11 [Promotion standards that equalize the abilities of marginal survivors] Suppose that at each stage $t$, the promotion standards $(m, f)$ maximize total ability net of wages (11a) (respectively, (11b)), so that mar- 
ginal male and female survivors have equal expected abilities. Suppose Assumption A holds.

(a) Whether or not the hierarchy has memory, if $m_{1}>0$, females face a lower promotion standard than males at stage 1 . That is, $m_{1}>f_{1}$.

(b) Suppose the hierarchy does not have memory. Then males eventually face lower promotion standards than females, but there are almost no marginal males. That is, given $\varepsilon>0$, there exists $\tilde{t}$ such that for $t>\tilde{t}, m_{t}<f_{t}$ and $\tilde{\phi}_{t}\left(m_{t}\right)<\varepsilon$.

(c) Suppose the hierarchy has memory, and that Assumption B holds. Then males eventually face the same promotion standards as females, but there are almost no marginal males. In particular, $\lim \sup m_{t}=\lim \sup f_{t}$. Given $\varepsilon>0$, there exists $\tilde{t}$ and $\delta>0$ such that for $t>\tilde{t}, \int_{-\infty}^{\bar{m}+\delta} g(a) \mathcal{S}_{t}^{M}(a, m) d a<$ $\varepsilon$.

Proof (a) It is enough to show that the expected ability of a marginal male is less than his promotion standard $m_{1}$. The expected ability of a marginal male can be written

$$
\begin{aligned}
e^{M}[A \mid m, 1] & =\int_{-\infty}^{\infty} a \frac{g(a) \phi^{u}\left(m_{1}-a\right)}{\int_{-\infty}^{\infty} g(a) \phi^{u}\left(m_{1}-a\right) d a} d a \\
& =\int_{-\infty}^{\infty}\left(m_{1}-x\right) \frac{g\left(m_{1}-x\right) \phi^{u}(x)}{\int_{-\infty}^{\infty} g(a) \phi^{u}\left(m_{1}-a\right) d a} d x \\
& =m_{1}-\int_{-\infty}^{\infty} x \frac{g\left(m_{1}-x\right) \phi^{u}(x)}{\int_{-\infty}^{\infty} g(a) \phi^{u}\left(m_{1}-a\right) d a} d x<m_{1}
\end{aligned}
$$

The inequality follows because the integral in (13) is positive. The denominator is positive, and the numerator can be written

$$
\begin{aligned}
& \int_{0}^{\infty} x g\left(m_{1}-x\right) \phi^{u}(x) d x+\int_{-\infty}^{0} x g\left(m_{1}-x\right) \phi^{u}(x) d x \\
& =\int_{0}^{\infty} y g\left(m_{1}-y\right) \phi^{u}(y) d y-\int_{0}^{\infty} y g\left(m_{1}+y\right) \phi^{u}(-y) d y \\
& =\int_{0}^{\infty} y\left[g\left(m_{1}-y\right)-g\left(m_{1}+y\right)\right] \phi^{u}(y) d y>0
\end{aligned}
$$


In the second line, $\phi^{u}(y)=\phi^{u}(-y)$ due to symmetry of $\phi^{u}$ and in the last line, $\left[g\left(m_{1}-y\right)-g\left(m_{1}+y\right)\right]>0$ due to $m_{1}>0$, symmetry of $g$, and single-peakedness of $g$.

(b) To show that $m_{t}<f_{t}$, let $f$ and $\bar{f}$ be the lower and upper bounds for $f$. Let $x \geq \bar{f}$. Using Lemma $3(\bar{d})$, if $m$ is bounded, then for large $t$, the expected ability of the marginal male survivor is larger than $x$, hence larger than $f_{t}$, a contradiction. Therefore $m$ is not bounded, and in particular, is not bounded below. Let $\tilde{t}$ be large enough that for $t>\tilde{t}, m_{t}<\underline{f} \leq f_{t}$.

I now show that $\tilde{\phi}_{t}\left(m_{t}\right)<\varepsilon$ for $t>\tilde{t}$. Using Assumption A, an upper bound for $\phi\left(m_{t} \mid a\right)=\phi^{u}\left(m_{t}-a\right)$ is $\phi^{u}(0)$. Let $\zeta, \gamma>0$ such that $\zeta \phi^{u}(0)+$ $\gamma<\varepsilon$.

Choose $x>0$ and $\tilde{t}$ large enough so that $\phi^{u}\left(m_{t}-a\right)<\phi^{u}(\bar{f}-a)<\gamma$ for $a>x$ and, using Lemma 3(b), so that $\int_{-\infty}^{x} \frac{g(a) S_{t}^{M}(a, m)}{\int_{-\infty}^{\infty} g(a) S_{t}^{M}(a, m) d a} d a<\zeta$. Then

$$
\begin{aligned}
\tilde{\phi}_{t}\left(m_{t}\right) & =\int_{-\infty}^{x} \phi\left(m_{t} \mid a\right) \frac{g(a) S_{t}^{M}(a, m)}{\int_{-\infty}^{\infty} g(a) S_{t}^{M}(a, m) d a} d a+\int_{x}^{\infty} \phi\left(m_{t} \mid a\right) \frac{g(a) S_{t}^{M}(a, m)}{\int_{-\infty}^{\infty} g(a) S_{t}^{M}(a, m) d a} d a \\
& <\int_{-\infty}^{x} \phi\left(m_{t} \mid a\right) \frac{g(a) S_{t}^{M}(a, m)}{\int_{-\infty}^{\infty} g(a) S_{t}^{M}(a, m) d a} d a+\gamma \int_{x}^{\infty} \frac{g(a) S_{t}^{M}(a, m)}{\int_{-\infty}^{\infty} g(a) S_{t}^{M}(a, m) d a} d a \\
& <\phi^{u}(0) \int_{-\infty}^{x} \frac{g(a) S_{t}^{M}(a, m)}{\int_{-\infty}^{\infty} g(a) S_{t}^{M}(a, m) d a} d a+\gamma<\phi^{u}(0) \zeta+\gamma<\varepsilon
\end{aligned}
$$

(c) The profit $\int_{\bar{m}}^{\infty}(a-\bar{w}) \overline{\mathcal{S}}^{M}(a, m) g(a) d a$ is maximized by choosing $\bar{m}=\bar{w}$, hence $\lim \sup m_{t}$ should be $\bar{m}$. Since $\limsup f_{t}=\bar{f}=\bar{w}$, the result follows. That $\int_{-\infty}^{\bar{m}+\varepsilon} g(a) \mathcal{S}_{t}^{M}(a, m) d a<\varepsilon$ for large $t$ follows from Lemma 7 and figure 2 .

When the promotion standards equalize the expected abilities of marginal survivors, both the survival rates and abilities of male and female survivors will differ. These differences have an efficiency explanation, and in particular do not follow from gender preferences on the part of employers.

However, disparate treatment due to a legitimate business objective is hard to distinguish from disparate treatment due to gender preferences. 
Suppose, for example, that firms choose promotion standards according to the following "discriminatory" objective:

$$
\operatorname{maximize}_{\left\{m_{t}, f_{t}\right\}} W_{t}(m, f)+U\left(\frac{E\left[\mathcal{S}_{t}^{M}(A, m)\right]}{E\left[\mathcal{S}_{t}^{F}(A, f)\right]}\right)
$$

If $U$ is increasing, the objective explicitly favors males. But the standard for males may still be higher at the beginning of the hierarchy than for females, and lower at the end, whether the objective is $W$ or $W+U$. Because of this ambiguity, courts and policymakers may resort to procedural rules such as a requirement for gender-blind standards or equal survival rates.

\subsection{Promotions within the firm: preserving options}

Suppose now that all promotions take place within a single firm. Then the firm itself profits from the information it collects through its promotion decisions. I show that the firm has an incentive to keep a male in the pool at early stages, despite a moderately bad signal, in order to collect more information and preserve an option. In particular, I show that at an early stage the firm will retain males with lower expected ability than will be tolerated in promoted females. In this sense, males are favored.

Consider a hierarchy with three stages $(t=0,1,2)$ and two promotions (at $t=1,2$ ). At stage 0 , there is no employer, but agents generate signals of ability. These are used for hiring in the first promotion stage. A firm's objective function is equal to the sum of workers' abilities in each promotion period, net of wages.

The firm's objective function is

$$
\begin{gathered}
\Pi\left(f_{1}, f_{2}, m_{1}, m_{2}\right)=\int_{f_{1}}^{\infty}\left(a-w_{1}\right) g(a) d a+\int_{\max \left\{f_{1}, f_{2}\right\}}^{\infty}\left(a-w_{2}\right) g(a) d a \\
+\int_{-\infty}^{\infty}\left(a-w_{1}\right) g(a)\left[1-\Phi\left(m_{1} \mid a\right)\right] d a+\int_{-\infty}^{\infty}\left(a-w_{2}\right) g(a)\left[1-\Phi\left(m_{1} \mid a\right)\right]\left[1-\Phi\left(m_{2} \mid a\right)\right] d a
\end{gathered}
$$

In Proposition 12(a), I assume that wages in both periods are equal. This 
assumption isolates the incentive to preserve options from the problem that the cost of a mistake is compounded when wages are increasing.

Proposition 12 [Preserving options within the firm] Suppose there are three periods with two promotions that take place within a single firm. Suppose the promotion standards $\left(f_{1}, f_{2}\right),\left(m_{1}, m_{2}\right)$ maximize (14).

(a) Suppose that $w_{1}=w_{2}=w$. Then the expected ability of a marginal male at the first promotion stage is smaller than the expected ability of a marginal female. That is, $e^{M}[A \mid m, 1]<f_{1}$.

(b) The expected ability of a marginal male at the final promotion stage is the same as that of a marginal female. That is, $e^{M}[A \mid m, 2]=f_{2}$.

Proof Since wages are nondecreasing, the optimum of (14) satisfies

$$
\begin{gathered}
f_{1}=w_{1} \\
f_{2}=w_{2} \\
\int_{-\infty}^{\infty}\left(a-w_{1}\right) g(a) \phi\left(m_{1} \mid a\right) d a+ \\
\int_{-\infty}^{\infty}\left(a-w_{2}\right) g(a) \phi\left(m_{1} \mid a\right)\left[1-\Phi\left(m_{2} \mid a\right)\right] d a=0 \\
\int_{-\infty}^{\infty}\left(a-w_{2}\right) g(a)\left[1-\Phi\left(m_{1} \mid a\right)\right] \phi\left(m_{2} \mid a\right) d a=0
\end{gathered}
$$

Write $\mathcal{M}_{1}$ for the probability of being a marginal male at the first promotion.

$$
\mathcal{M}_{1}=\int_{-\infty}^{\infty} g(a) \phi\left(m_{1} \mid a\right) d a=\int_{-\infty}^{\infty} g(\alpha+w) \phi\left(m_{1} \mid \alpha+w\right) d \alpha
$$

Write $\mathcal{M}_{2}$ for the probability of being a marginal male in the first promotion and also surviving the second promotion.

$$
\begin{aligned}
\mathcal{M}_{2} & =\int_{-\infty}^{\infty} g(a) \phi\left(m_{1} \mid a\right)\left[1-\Phi\left(m_{2} \mid a\right)\right] d a \\
& =\int_{-\infty}^{\infty} g(\alpha+w) \phi\left(m_{1} \mid \alpha+w\right)\left[1-\Phi\left(m_{2} \mid \alpha+w\right)\right] d \alpha
\end{aligned}
$$


Then the first-order condition (17) can be written

$$
\begin{gathered}
\mathcal{M}_{1} \int_{-\infty}^{\infty} \alpha \frac{g(\alpha+w) \phi\left(m_{1} \mid \alpha+w\right)}{\mathcal{M}_{1}} d \alpha+ \\
\mathcal{M}_{2} \int_{-\infty}^{\infty} \alpha \frac{g(\alpha+w) \phi\left(m_{1} \mid \alpha+w\right)\left[1-\Phi\left(m_{2} \mid \alpha+w\right)\right]}{\mathcal{M}_{2}} d \alpha=0
\end{gathered}
$$

The density in the first integral of (20) describes the males who were marginal at the first promotion. The density in the second integral of (20) describes the males remaining from that group after the second promotion. Because $\Phi\left(m_{2} \mid \alpha+w\right)$ is decreasing with $\alpha$, the distribution in the second integral stochastically dominates the distribution in the first integral. Because of stochastic dominance, the expected ability represented by the first integral is smaller than the expected ability represented by the second integral. Since the two terms sum to zero, the first integral is negative and the second is positive. That is,

$$
\begin{aligned}
& \int_{-\infty}^{\infty}(a-w) \frac{g(a) \phi\left(m_{1} \mid a\right)}{\int_{-\infty}^{\infty} g(a) \phi\left(m_{1} \mid a\right) d a} d a< \\
& \quad 0<\int_{-\infty}^{\infty}(a-w) \frac{g(a) \phi\left(m_{2} \mid a\right)\left[1-\Phi\left(m_{2} \mid a\right)\right]}{\int_{-\infty}^{\infty} g(a) \phi\left(m_{2} \mid a\right)\left[1-\Phi\left(m_{2} \mid a\right)\right] d a} d a
\end{aligned}
$$
(a).

Using (15), the first inequality implies $e^{M}[A \mid m, 1]<w=f_{1}$. This proves

Part (b) follows from (16), which says $f_{2}=w_{2}$, and from (18), which says $e^{M}[A \mid m, 2]=w_{2}$.

When promotions are within the firm, one cannot say in general whether males will be promoted at a lower or higher standard than females at the first stage, but conditional on a given standard for females, more males will be promoted within the firm than if promotions were across firms. Again, this is because the firm internalizes the information it generates by keeping males in the pool.

Finally, Proposition 12 also describes the optimal policy when the firm's profit depends on the signals produced by the workers rather than on their 
abilities. For example, the value produced in an academic labor market is the quality of the papers written, rather than the ability of the professor who produced them. The objective function would then be

$$
\begin{aligned}
& \Pi^{z}\left(f_{1}, f_{2}, m_{1}, m_{2}\right)=\int_{f_{1}}^{\infty}\left(a-w_{1}\right) g(a) d a+\int_{f_{2}}^{\infty}\left(a-w_{1}\right) g(a) d a \\
& \quad+\int_{-\infty}^{\infty} \int_{-\infty}^{\infty}\left(z_{1}-w_{1}\right) g(a)\left[1-\Phi\left(m_{1} \mid a\right)\right] \phi\left(z_{1} \mid a\right) d z_{1} d a \\
& \quad+\int_{-\infty}^{\infty} \int_{-\infty}^{\infty}\left(z_{2}-w_{1}\right) g(a)\left[1-\Phi\left(m_{1} \mid a\right)\right]\left[1-\Phi\left(m_{2} \mid a\right)\right] \phi\left(z_{2} \mid a\right) d z_{2} d a
\end{aligned}
$$

But, integrating on $z_{1}$ and $z_{2}$, since $\int_{-\infty}^{\infty} z \phi(z \mid a) d z=a$, it holds that $\Pi^{z}\left(f_{1}, f_{2}, m_{1}, m_{2}\right)=\Pi\left(f_{1}, f_{2}, m_{1}, m_{2}\right)$.

\section{Conclusion}

Affirmative action policies have been justified and evaluated from many different perspectives, on both efficiency grounds and equity grounds. For the most part, economists have focussed on efficiency, especially productive efficiency. For example, Holzer and Neumark (2000) argue from an extensive empirical literature that "affirmative action offers significant redistribution toward women and minorities, with relatively small efficiency consequences" (page 559). Among the ingenious theoretical arguments for why affirmative action policies enhance efficiency are those of Lundberg and Startz (1983) and Lundberg (1991), who consider a model of statistical discrimination where wages depend on imperfect signals of ability. They show, among other things, that the pooling of workers with different signaling ability creates more incentive to invest in human capital. Milgrom and Oster (1987) argue that affirmative action policies can efficiently prevent employers from underpromoting women and minorities. The incentive to underpromote derives from a fear of revealing the worth of their employees to rival firms, a threat which is higher for the more "invisible" workers, such as women and minorities.

I have not made efficiency central to my analysis, since it is unclear how to define it in the context studied here. I have taken the social objectives of gender blindness or equal promotions as primitive, rather than deriving those 
objectives from a welfare function. This has allowed me to illuminate conflicts among those objectives that derive from gender differences in behavior, in particular, risk taking. I have simply assumed that males are hard-wired for risk taking and females are not, and that all agents want to stay in the hierarchy if allowed.

The efficiency criterion that springs to mind for most economists is that marginal survivors should have the same expected ability. This is the criterion that drives promotion standards when promotions are across firms, but only because the employers do not internalize the benefits of collecting information on risk takers, which can possibly create a better pool for the long run. When promotions are within firms, the firm has an incentive to preserve an option on males at the beginning, since more information will be collected later. The firm itself profits from this information. Thus, the promotion standards will be such that the marginal male has lower expected ability than the marginal female, and in this sense, males are favored. However, the promotion standard itself may be higher for males than for females in both cases.

There are many other gender differences at work as well. Some of these differences can be confounding factors that overturn any intrinsic difference in risk taking. In particular, Becker and Eagly (2004) found that females were at least as likely as males to put themselves at risk in protecting Jews in the Holocaust, and females are considerably more likely to put themselves at risk by donating kidneys to relatives in need. However, the authors hypothesize that such behavior might be rooted in a greater willingness of females to care for others, or to heed an ethical calling. Females may be motivated by objectives that overcome, and therefore obscure, an aversion to risk.

Further, as I mentioned in the introduction, the noisiness of the signals is not entirely under the control of the employee. Even if males are more risktaking, they may be more closely observed within each promotion period than females. The latter hypothesis is consonant with the Milgrom and Oster (1987) "invisibility" hypothesis. If that effect is strong enough, then females should be understood as the workers who present noisy signals, and the males should be understood as generating more accurate signals.

In any case, the main point of the paper is that gender differences in tastes set up a conflict between procedural fairness and disparate impacts. 
Differences in risk taking are one example of that larger point. ${ }^{11}$ A legitimate business purpose is a defense to disparate impact, but as mentioned in section 5, a legitimate business purpose cannot always be distinguished from discriminatory intent.

\section{Appendix}

\section{Proof of Lemma 1}

The same argument applies whether the survival rates are calculated with or without memory. Arguing for the hierarchy without memory, it holds that

$$
\begin{aligned}
& \int_{-\infty}^{\infty} a\left[\frac{\mathcal{S}_{t}^{M}(a, m)}{E\left[\mathcal{S}_{t}^{M}(A, m)\right]}-\frac{\mathcal{S}_{t}^{F}(a, f)}{E\left[\mathcal{S}_{t}^{F}(A, f)\right]}\right] g(a) d a \\
& =\int_{-\infty}^{\infty}\left(a-f_{t}\right)\left[\frac{\mathcal{S}_{t}^{M}(a, m)}{E\left[\mathcal{S}_{t}^{M}(A, m)\right]}-\frac{\mathcal{S}_{t}^{F}(a, f)}{E\left[\mathcal{S}_{t}^{F}(A, f)\right]}\right] g(a) d a \\
& =\int_{-\infty}^{f_{t}}\left(a-f_{t}\right)\left[\frac{\mathcal{S}_{t}^{M}(a, m)}{E\left[\mathcal{S}_{t}^{M}(A, m)\right]}-\frac{\mathcal{S}_{t}^{F}(a, f)}{E\left[\mathcal{S}_{t}^{F}(A, f)\right]}\right] g(a) d a \\
& +\int_{f_{t}}^{\infty}\left(a-f_{t}\right)\left[\frac{\mathcal{S}_{t}^{M}(a, m)}{E\left[\mathcal{S}_{t}^{M}(A, m)\right]}-\frac{\mathcal{S}_{t}^{F}(a, f)}{E\left[\mathcal{S}_{t}^{F}(A, f)\right]}\right] g(a) d a \\
& <0
\end{aligned}
$$

In the third line, where $\left(a-f_{t}\right)<0$, it holds that $\mathcal{S}_{t}^{F}(a, f)=0$ and $0<\mathcal{S}_{t}^{M}(a, m)<1$. Therefore, $\frac{\mathcal{S}_{t}^{M}(a, m)}{E\left[\mathcal{S}_{t}^{M}(A, m)\right]}-\frac{\mathcal{S}_{t}^{F}(a, m)}{E\left[\mathcal{S}_{t}^{F}(A, f)\right]}>0$, so the integrand is negative. In the fourth line, where $\left(a-f_{t}\right) \geq 0$, it holds that $0<\mathcal{S}_{t}^{M}(a, m)<1=\mathcal{S}_{t}^{F}(a, f)$. Since $E\left[\mathcal{S}_{t}^{M}(A, m)\right] \geq E\left[\mathcal{S}_{t}^{F}(A, f)\right]$, it holds that $\frac{\mathcal{S}_{t}^{M}(a, m)}{E\left[\mathcal{S}_{t}^{M}(A, m)\right]}<\frac{\mathcal{S}_{t}^{F}(a, f)}{E\left[\mathcal{S}_{t}^{F}(A, f)\right]}$. Therefore the integrand is again negative. Then

$$
\begin{aligned}
0 & >\int_{-\infty}^{\infty}\left(a-f_{t}\right)\left[\frac{\mathcal{S}_{t}^{M}(a, m)}{E\left[\mathcal{S}_{t}^{M}(A, m)\right]}-\frac{\mathcal{S}_{t}^{F}(a, f)}{E\left[\mathcal{S}_{t}^{F}(A, f)\right]}\right] g(a) d a \\
& =\int_{-\infty}^{\infty} a\left[\frac{\mathcal{S}_{t}^{M}(a, m)}{E\left[\mathcal{S}_{t}^{M}(A, m)\right]}-\frac{\mathcal{S}_{t}^{F}(a, f)}{E\left[\mathcal{S}_{t}^{F}(A, f)\right]}\right] g(a) d a
\end{aligned}
$$

\footnotetext{
${ }^{11}$ In a model where the signal for promotion depends on effort, Bardsley and Sherstyuk (2006) show that differences in the cost of effort can also lead to different promotion rates.
} 


\section{Proof of Lemma 3}

Let $\underline{m} \leq m_{t} \leq \bar{m}$ for all $t=1,2, \ldots$ Since the distributions $G$ and $\Phi$ have full support, the probability that any male survives at any date $t$, conditional

on having survived to $t-1$, is strictly less than one. That is, $1-\Phi\left(m_{t} \mid a\right)<1$ for every $t$ and every $a \in \mathbf{R}$.

(a) Let $\varepsilon>0$. Let $\tilde{a}>0$ satisfy $0<1-G(\tilde{a})<\varepsilon / 2$ and let $\tilde{t}$ satisfy $(1-\Phi(\underline{m} \mid a))^{\tilde{t}}<\varepsilon / 2$ for all $a \leq \tilde{a}$. Then for $t \geq \tilde{t}$,

$$
\begin{aligned}
& \int_{-\infty}^{\infty} g(a) \mathcal{S}_{t}^{M}(a, m) d a \\
& =\int_{-\infty}^{\tilde{a}} g(a) \Pi_{d=1}^{t}\left(1-\Phi\left(m_{d} \mid a\right)\right) d a+\int_{\tilde{a}}^{\infty} g(a) \Pi_{d=1}^{t}\left(1-\Phi\left(m_{d} \mid a\right)\right) d a \\
& \leq \int_{-\infty}^{\tilde{a}} g(a)(1-\Phi(\underline{m} \mid a))^{t} d a+\int_{\tilde{a}}^{\infty} g(a) \Pi_{d=1}^{t}\left(1-\Phi\left(m_{d} \mid a\right)\right) d a \\
& <G(\tilde{a}) \varepsilon / 2+(1-G(\tilde{a})<\varepsilon
\end{aligned}
$$

(b) Given $x \in \mathbf{R}$, the probability that a random male survivor has ability greater than $x$ is

$$
\begin{aligned}
& \frac{\int_{x}^{\infty} g(a) \mathcal{S}_{t}^{M}(a, m) d a}{\int_{x}^{\infty} g(a) \mathcal{S}_{t}^{M}(a, m) d a+\int_{-\infty}^{x} g(a) \mathcal{S}_{t}^{M}(a, m) d a} \\
& >\frac{\int_{x}^{\infty} g(a) \mathcal{S}_{t}^{M}(a, m) d a}{\int_{x}^{\infty} g(a) \mathcal{S}_{t}^{M}(a, m) d a+\mathcal{S}_{t}^{M}(x, m) \int_{-\infty}^{x} g(a) d a} \\
& =\frac{\int_{x}^{\infty} g(a) \frac{\mathcal{S}_{t}^{M}(a, m)}{\mathcal{S}_{t}^{M}(x, m)} d a}{\int_{x}^{\infty} g(a) \frac{\mathcal{S}_{t}^{M}(a, m)}{\mathcal{S}_{t}^{M}(x, m)} d a+G(x)}
\end{aligned}
$$

Using the boundedness of $m$, and the fact that $\Phi\left(m_{d} \mid a\right)$ decreases with $a$ due to the monotone likelihood ratio property, it holds as $t$ gets large that

$$
\frac{\mathcal{S}_{t}^{M}(a, m)}{\mathcal{S}_{t}^{M}(x, m)}=\prod_{d=1}^{t} \frac{\left[1-\Phi\left(m_{d} \mid a\right)\right]}{\left[1-\Phi\left(m_{d} \mid x\right)\right]} \rightarrow \infty \text { for } a>x
$$


Thus, (22) converges to one as $t$ becomes large. The result follows.

(c) For given $t$, write the expected ability of surviving males, $E^{M}[A \mid m, t]$, in two parts. The first term in (24) is the expected ability of survivors who satisfy $|A|>4 x$, times the probability of that event, and the second part is the expected ability of survivors who satisfy $|A| \leq 4 x$, times the probability of that event.

$E^{M}[A|m, t| A \mid,>4 x] \times \operatorname{Pr}[|A|>4 x]+E^{M}[A|m, t| A \mid, \leq 4 x] \times \operatorname{Pr}[|A| \leq 4 x]$

or equivalently,

$$
\begin{aligned}
E^{M}[A \mid m, t] & =\left[\frac{\int_{4 x}^{\infty} a g(a) \mathcal{S}_{t}^{M}(a, m) d a+\int_{-\infty}^{-4 x} a g(a) \mathcal{S}_{t}^{M}(a, m) d a}{\int_{-\infty}^{\infty} g(a) \mathcal{S}_{t}^{M}(a, m) d a}\right]+\frac{\int_{-4 x}^{4 x} a g(a) \mathcal{S}_{t}^{M}(a, m) d a}{\int_{-\infty}^{\infty} g(a) \mathcal{S}_{t}^{M}(a, m) d a} \\
& =\left[\frac{\int_{4 x}^{\infty} a g(a) \mathcal{S}_{t}^{M}(a, m) d a+\int_{-\infty}^{-4 x} a g(a) \mathcal{S}_{t}^{M}(a, m) d a}{\int_{4 x}^{\infty} g(a) \mathcal{S}_{t}^{M}(a, m) d a+\int_{-\infty}^{-4 x} g(a) \mathcal{S}_{t}^{M}(a, m) d a}\right] \\
& \times\left[\frac{\int_{4 x}^{\infty} g(a) \mathcal{S}_{t}^{M}(a, m) d a+\int_{-\infty}^{-4 x} g(a) \mathcal{S}_{t}^{M}(a, m) d a}{\int_{-\infty}^{\infty} g(a) \mathcal{S}_{t}^{M}(a, m) d a}\right] \\
& +\left[\frac{\int_{-4 x}^{4 x} a g(a) \mathcal{S}_{t}^{M}(a, m) d a}{\int_{-4 x}^{4 x} g(a) \mathcal{S}_{t}^{M}(a, m) d a}\right] \times\left[\frac{\int_{-4 x}^{4 x} g(a) \mathcal{S}_{t}^{M}(a, m) d a}{\int_{-\infty}^{\infty} g(a) \mathcal{S}_{t}^{M}(a, m) d a}\right]
\end{aligned}
$$

Since $\operatorname{Pr}[|A|>4 x]+\operatorname{Pr}[|A| \leq 4 x]=1$, and $E^{M}[A|m, t| A \mid, \leq 4 x]>-4 x$, it will be enough to show that as $t$ becomes large, $E^{M}[A|m, t| A \mid,>4 x]$ becomes large (larger than $2 x$ ) and $\operatorname{Pr}[|A| \leq 4 x]$ becomes small (smaller than 1/6). Then $E^{M}[A \mid m, t]>(2 x)(5 / 6)-(4 x)(1 / 6)=x$.

First show that $E^{M}[A|m, t| A \mid,>4 x]>2 x$ for large $t$.

$$
\begin{aligned}
& E^{M}[A|m, t,| A \mid>4 x] \\
& =\frac{\int_{4 x}^{\infty} a g(a) \mathcal{S}_{t}^{M}(a, m) d a+\int_{-\infty}^{-4 x} a g(a) \mathcal{S}_{t}^{M}(a, m) d a}{\int_{4 x}^{\infty} g(a) \mathcal{S}_{t}^{M}(a, m) d a+\int_{-\infty}^{-4 x} g(a) \mathcal{S}_{t}^{M}(a, m) d a} \\
& >\frac{4 x \int_{4 x}^{\infty} g(a) \mathcal{S}_{t}^{M}(a, m) d a+\int_{-\infty}^{-4 x} a g(a) \mathcal{S}_{t}^{M}(a, m) d a}{\int_{4 x}^{\infty} g(a) \mathcal{S}_{t}^{M}(a, m) d a+\mathcal{S}_{t}^{M}(-4 x, m) G(-4 x)} \\
& =\left[4 x+\frac{\int_{-\infty}^{-4 x} a g(a) \frac{\mathcal{S}_{t}^{M}(a, m)}{\mathcal{S}_{t}^{M}(-4 x, m)} d a}{\int_{4 x}^{\infty} g(a) \frac{\mathcal{S}_{t}^{M}(a, m)}{\mathcal{S}_{t}^{M}(-4 x, m)} d a}\right] /\left[1+\frac{G(-4 x)}{\int_{4 x}^{\infty} g(a) \frac{\mathcal{S}_{t}^{M}(a, m)}{\mathcal{S}_{t}^{M}(-4 x, m)} d a}\right]
\end{aligned}
$$


Using the boundedness of $m$, and the fact that $\Phi\left(m_{d} \mid a\right)$ decreases with $a$ due to the monotone likelihood ratio property, it holds as $t$ gets large that

$$
\begin{aligned}
& \frac{\mathcal{S}_{t}^{M}(a, m)}{\mathcal{S}_{t}^{M}(-4 x, m)}=\prod_{d=1}^{t} \frac{\left[1-\Phi\left(m_{d} \mid a\right)\right]}{\left[1-\Phi\left(m_{d} \mid-4 x\right)\right]} \rightarrow 0 \text { for } a<-4 x \\
& \frac{\mathcal{S}_{t}^{M}(a, m)}{\mathcal{S}_{t}^{M}(-4 x, m)}=\prod_{d=1}^{t} \frac{\left[1-\Phi\left(m_{d} \mid a\right)\right]}{\left[1-\Phi\left(m_{d} \mid-4 x\right)\right]} \rightarrow \infty \text { for } a>4 x
\end{aligned}
$$

The value of $(25)$ is less than $4 x$, since the numerator adds a negative term to $4 x$, and the denominator of $(25)$ is greater than one. However, using the fact that $\int_{-\infty}^{-4 x} a g(a) d a$ is finite ${ }^{12}$ and using (26),(27), it follows that the negative term in the numerator vanishes for large enough $t$. Using (27), the second term of the denominator of (25) vanishes as $t$ becomes large, and we can therefore assert that $E^{M}[A|m, t| A \mid,>4 x]>2 x$ for large enough $t$.

I now show that $\operatorname{Pr}[|A| \leq 4 x]<1 / 6$ for large enough $t$.

$$
\begin{aligned}
\operatorname{Pr}[|A| \leq 4 x] & =\frac{\int_{-4 x}^{4 x} g(a) \mathcal{S}_{t}^{M}(a, m) d a}{\int_{-\infty}^{\infty} g(a) \mathcal{S}_{t}^{M}(a, m) d a}<\frac{\int_{-4 x}^{4 x} g(a) \mathcal{S}_{t}^{M}(4 x, m) d a}{\int_{-\infty}^{\infty} g(a) \mathcal{S}_{t}^{M}(a, m) d a} \\
& =\frac{\int_{-4 x}^{4 x} g(a) d a}{\int_{-\infty}^{\infty} g(a) \frac{\mathcal{S}_{t}^{M}(a, m)}{\mathcal{S}_{t}^{M}(4 x, m)} d a}=\frac{\int_{-4 x}^{4 x} g(a) d a}{\int_{-\infty}^{4 x} g(a) \frac{\mathcal{S}_{t}^{M}(a, m)}{\mathcal{S}_{t}^{M}(4 x, m)} d a+\int_{4 x}^{\infty} g(a) \frac{\mathcal{S}_{t}^{M}(a, m)}{\mathcal{S}_{t}^{M}(4 x, m)} d a}
\end{aligned}
$$

The result follows from (23), substituting $4 x$ for $x$, since the denominator becomes large.

(d) I omit the proof, which is essentially the same as for part (c), replacing $E^{M}[A \mid m, t]$ with $e^{M}[A \mid m, t]$, and using the fact that $\phi$ is bounded.

\section{Proof of Lemma 7}

The sequence $c=c_{1}, c_{2}, \ldots c_{t}, \ldots$ contains at most a finite number of values larger than $\bar{c}+\varepsilon$. Let $\bar{t}$ be the largest value of $t$ in this set, that is, the latest date such that $c_{t}>\bar{c}+\varepsilon$. (If the sequence is increasing as in Proposition 8, one can take $\varepsilon=0$ and $\bar{t}=0$.) Because $\phi$ has full support, there is a positive probability of survival to time $\bar{t}$, regardless of ability, that is, $\overline{\mathcal{S}}_{\bar{t}}^{M}(a, c)>0$ for all $a$.

\footnotetext{
${ }^{12}$ Notice that $\int_{-\infty}^{\infty} a^{2} g(a) d a$ is finite because variance is finite.
} 
That $\overline{\mathcal{S}}_{t}^{M}(a, c) \rightarrow 0$ for $a<\bar{c}$ follows because $\bar{Z}^{t}$ converges in probability to $a$ and $a<\bar{c}$.

That $\overline{\mathcal{S}}_{t}^{M}(\bar{c}, c) \rightarrow 0$ follows from the central limit theorem. When $a=\bar{c}$, the limit distribution of $\sqrt{t}\left(\bar{Z}^{t}-\bar{c}\right) / v$ is normal, centered at 0 , where $v^{2}$ is the variance of $\Phi$. With positive probability it holds that $\sqrt{t}\left(\bar{Z}^{t}-\bar{c}\right) / v<0$. But since survival requires that $\bar{Z}^{t}-\bar{c} \geq 0$ for all $t=1,2, \ldots$, the joint probability of survival at $t=1,2, \ldots$ falls to zero.

I now show that for $a>\bar{c}+\varepsilon, \overline{\mathcal{S}}^{M}(a, c)>0$.

For arbitrary dates $t_{1}, t_{2}$, it is useful to introduce the notation $\bar{Z}_{t_{1}}^{t_{2}}$ as the average of signals accrued between those dates, namely $\bar{Z}_{t_{1}}^{t_{2}}=\frac{1}{t_{2}-t_{1}} \sum_{k=t_{1}+1}^{t_{2}} Z_{k}$. The previous notation $\bar{Z}^{t}$ becomes $\bar{Z}_{0}^{t}$.

Let $\mathcal{P}(a, c ; \bar{t}, Z)$ be the probability that an agent with ability $A=a$ survives in the limit, conditional on having survived to $\bar{t}$ with average signal $\bar{Z}_{0}^{\bar{t}}=Z \in \mathbf{R}$. Survival implies that $Z \geq c_{\bar{t}}$. Given $a$ and $Z$, write

$$
\mathcal{P}(a, c ; \bar{t}, Z)=\operatorname{Pr}\left[\left(\begin{array}{l}
\bar{t} \\
\bar{t}
\end{array}\right) Z+\left(\frac{t-\bar{t}}{t}\right) \bar{Z}_{\bar{t}}^{t} \geq c_{t} \text { for all } t=\bar{t}+1, \bar{t}+2, \ldots\right]
$$

where $\left\{\bar{Z}_{\bar{t}}^{t}: t=\bar{t}+1, \bar{t}+2, \ldots\right\}$ is the random variable.

It is enough to show that $\mathcal{P}(a, c ; \bar{t}, Z)>0$ for all $a>\bar{c}+\varepsilon$ and all $Z \geq c_{\bar{t}}$. Since $\phi$ has full support, $\overline{\mathcal{S}}_{\bar{t}}^{M}(a, c)>0$ for all abilities $a$. Therefore if $\mathcal{P}(a, c ; \bar{t}, Z)>0$ for all $a>\bar{c}+\varepsilon$ and $Z \geq c_{\bar{t}}$, it holds that $\overline{\mathcal{S}}^{M}(a, c)>0$ for $a>\bar{c}+\varepsilon$.

The first line below follows from (28), because $c_{\bar{t}}>c_{t}$, hence, using $Z \geq c_{\bar{t}}>c_{t}$, it holds that $Z \geq c_{t}$ for all $t>\bar{t}$. Therefore the event $\left[\bar{Z}_{\bar{t}}^{t} \geq c_{t}\right.$ for all $\left.t=\bar{t}+1, \bar{t}+2, \ldots\right]$ implies the event $\left[\left(\frac{\bar{t}}{t}\right) Z+\left(\frac{t-\bar{t}}{t}\right) \bar{Z}_{\bar{t}}^{t} \geq c_{t}\right.$ for all $t=\bar{t}+1, \bar{t}+2, .$. but not vice versa.

The second line is a change of notation. In the third line, the constant value $a$ is subtracted from both sides of the inequality. The fourth line uses the fact that $c_{\tau+\bar{t}} \leq \bar{c}+\varepsilon$ for all $\tau>0$. The fifth line follows because the 
distribution of signals is atomless.

$$
\begin{aligned}
\mathcal{P}(a, c ; \bar{t}, Z) & >\operatorname{Pr}\left[\bar{Z}_{\bar{t}}^{t} \geq c_{t} \text { for all } t=\bar{t}+1, \bar{t}+2, \ldots\right] \\
& =\operatorname{Pr}\left[\bar{Z}_{0}^{\tau} \geq c_{\tau+\bar{t}} \text { for all } \tau=1,2, \ldots\right] \\
& =\operatorname{Pr}\left[\bar{Z}_{0}^{\tau}-a \geq c_{\tau+\bar{t}}-a \text { for all } \tau=1,2, \ldots\right] \\
& \geq \operatorname{Pr}\left[\bar{Z}_{0}^{\tau}-a \geq \bar{c}+\varepsilon-a \text { for all } \tau=1,2, \ldots\right] \\
& =\operatorname{Pr}\left[\bar{Z}_{0}^{\tau}-a>\bar{c}+\varepsilon-a \text { for all } \tau=1,2, \ldots\right]
\end{aligned}
$$

For $a>c+\varepsilon$, choose $b_{1}, b_{2}>0$ so that $-b_{1} v^{2}-b_{2}=\bar{c}+\varepsilon-a$. Then

$$
\begin{aligned}
\mathcal{P}(a, c ; \bar{t}, Z) & >\operatorname{Pr}\left[\bar{Z}_{0}^{\tau}-a>\bar{c}+\varepsilon-a \text { for all } \tau=1,2, \ldots\right] \\
& =\operatorname{Pr}\left[\bar{Z}_{0}^{\tau}-a>-b_{1} v^{2}-b_{2} \text { for all } \tau=1,2, \ldots\right] \\
& \geq \operatorname{Pr}\left[\bar{Z}_{0}^{\tau}-a>-b_{1} v^{2}-b_{2} / \tau \text { for all } \tau=1,2, \ldots\right]
\end{aligned}
$$

Using Lemma (6) of Dubins and Freedman (1965, p. 801),

$$
\operatorname{Pr}\left[\bar{Z}_{0}^{\tau}-a \leq-b_{1} v^{2}-\frac{b_{2}}{\tau} \text { for some } \tau=1,2, \ldots\right] \leq \frac{1}{1+b_{1} b_{2}}
$$

Thus,

$$
\begin{aligned}
& \mathcal{P}(a, c ; \bar{t}, Z) \\
& >\operatorname{Pr}\left[\bar{Z}_{0}^{\tau}-a>-b_{1} v^{2}-\frac{b_{2}}{\tau} \text { for all } \tau=1,2, \ldots\right] \geq 1-\frac{1}{1+b_{1} b_{2}}>0
\end{aligned}
$$




\section{References}

[1] Bajtelsmit, Vickie L. and Alexandra Bernasek, (1996), "Why do Women Invest Differently than Men?" Financial Counseling and Planning, 7, 110 .

[2] Bardsley, Peter and Sherstyuk, Katerina (2006), "Rat Races and Glass Ceilings." Topics in Theoretical Economics, 6 , Article 13. Available at: http://www.bepress.com/bejte/topics/vol6/iss1/art13

[3] Becker, Selwyn W. and Alice H. Eagly, (2004), "The Heroism of Women and Men." American Psychologist, 59, 163-178.

[4] Blumrosen, Alfred W., (1993), Modern Law: The Law Transmission System and Equal Employment Opportunity. Madison: University of Wisconsin Press.

[5] Charness, Gary and Uri Gneezy, (2004), "Gender Differences in Financial Risk Taking." Mimeograph. University of California, Santa Barbara.

[6] Dekel, E. and Suzanne Scotchmer, (1999), "On the Evolution of Attitudes Towards Risk in Winner-Take-All Games." Journal of Economic Theory, 87, 125-143.

[7] Dubins, L. E. and D. A. Freedman, (1965), "A Sharper Form of the Borel-Cantelli Lemma and the Strong Law." Annals of Mathematical Statistics, 23, 493-507.

[8] Eckel, Catherine C. and Philip J. Grossman, (2002), "Sex differences and statistical stereotyping in attitudes toward financial risk." Evolution and Human Behavior, 23, 281-295.

[9] Eckel, Catherine C. and Philip J. Grossman, (2005a), "Sex and Risk: Experimental Evidence." (Forthcoming in) Handbook of Experimental Economics Results. Amsterdam: Elsevier Science (North-Holland).

[10] Edley, Jr., Christopher, (1996), Not All Black and White: Affirmative Action, Race and American Values. New York: Hill and Wang. 
[11] Espinosa, Maria Paz and Gardeazabal, Javier, (2005), "Do Students Behave Rationally in Multiple-Choice Tests? Evidence from a Field Experiment." Mimeograph, Universidad del Pais Vasco.

[12] Ginsburg, Harvey. J. and Kimberly Miller, (2002), "Sex differences in children's risk taking." Child Development, 53, 426-428.

[13] Griggs v. Duke Power Co. 401 U.S. 424 (1971).

[14] Harry J. Holzer and David Neumark, (2000), "Assessing Affirmative Action." Journal of Economic Literature, XXXVIII, 483-569.

[15] Harry J. Holzer and David Neumark, (2006), "Equal Employment Opportunities and Affirmative Action." In William M. Rodgers III, ed., Handbook on the Economics of Discrimination, 260-287. Northampton, MA: Edward Elgar Publishers.

[16] Jianakoplos, N. A. and Alexandra Bernasek, (1998), "Are Women More Risk Averse?" Economic Inquiry, 36, 620-630.

[17] Krieger, Linda Hamilton, (1995), "The Content of Our Categories: A Cognitive Bias Approach to Discrimination and Equal Employment Opportunity." Stanford Law Review, 47, 1161-1248.

[18] Leiter, Samuel and Leiter, William M., (2002), Affirmative Action in Antidisrimination Law and Policy. Albany: State Universtiy of New York Press.

[19] Lundberg, Shelly J., (1991), "The Enforcement of Equal Opportunity Laws under Imperfect Information: Affirmative Action and Alternatives." Quarterly Journal of Economics, 106, 309-26.

[20] Shelly J. Lundberg and Richard Startz, (1983), "Private Discrimination and Social Intervention in Competitive Labor Markets." American Economic Review, 73, 340-347.

[21] Paul Milgrom and Sharon Oster, (1987), "Job Discrimination, Market Forces and the Invisibility Hypothesis." Quarterly Journal of Economics, 102, 453-476. 
[22] Mittal, Manish and Dhade, Aruna, (2007), "Gender Difference in Investment Risk-Taking: An Empirical Study." Icfai Journal of Behavioral Finance, 4, 32-42.

[23] Rodgers III, William M., ed, (2006), Handbook on the Economics of Discrimination, 260-287. Northampton, MA: Edward Elgar Publishers.

[24] Slovic, Paul, (1966), "Risk-Taking in Children: Age and Sex Differences." Child Development, 37, 169-176.

[25] Sobel, Joel, (2001), "On the Dynamics of Standards." RAND Journal of Economics, 32, 606-623.

[26] Spitzer, Matthew L., (1979), "Multicriteria choice processes: an application of public choice theory to Bakke, the FCC , and the courts." Yale Law Journal, 88, 717-779.

[27] University of California v. Bakke, 438 U.S. 265 (1978). 